\title{
Magnetic Resonance Imaging Grading of Pituitary Macroadenoma- SIPAP Classification Revisited.
}

\section{Shayan Sirat Maheen Anwar}

Aga Khan University Hospital

Kiran Hilal

Aga Khan University Hospital

Anam Khan ( $\nabla$ anam.khan@aku.edu )

Aga Khan University Hospital

\section{Asra Ahmad}

Aga Khan University Hospital

\section{Research Article}

Keywords: Magnetic resonance imaging, pituitary adenoma, tumor volume, SIPAP classification, Knosp grading.

Posted Date: October 14th, 2021

DOl: https://doi.org/10.21203/rs.3.rs-955034/v1

License: (1) (1) This work is licensed under a Creative Commons Attribution 4.0 International License. Read Full License 


\section{Abstract}

\section{BACKGROUND}

Magnetic resonance imaging (MRI) is regarded as the modality of choice in diagnosis of pituitary adenomas. Since surgery is the first line therapy for all pituitary adenomas with exception of prolactinoma, simple and reproducible MRI classification based on major directions of tumour growth is an essential tool providing the exact road map for right surgical approach and maximum safe resection.

SIPAP MRI classification for pituitary adenomas is derived from KNOSP-STEINER classification of parasellar growth, to which has been added grading for superior, inferior, anterior and posterior tumour extension.

We, therefore, propose to incorporate SIPAP classification in routine reporting template of pituitary adenomas, for ideal radiological description of tumour delineation, relationship to juxtasellar structures, and tumour size, hence facilitating greater success rate in surgical and subsequent clinical management.

\section{METHODS}

Two radiologists retrospectively reviewed imaging of 49 patients with biopsy-proven pituitary macroadenoma and graded according to SIPAP classification. Study was conducted at Aga Khan University Hospital from 1st July 2019 to $30^{\text {th }}$ June 2020. Data was analyzed using Stata version 15. Interobserver variability was calculated using Cohen's Kappa. Comparison between grading before and after treatment was performed by Chi-square test. $P$ values $<0.05$ were considered statistically significant.

\section{RESULTS}

Sixty three percent patients were male and $37 \%$ female. Overall, maximum preoperative and postoperative volume was $71.82 \mathrm{~cm}^{3}$ and $49.50 \mathrm{~cm}^{3}$ respectively, with significant difference in pre and post-operative volumes $(14.1 \pm 17.7$ vs. $4.5 \pm 10.4$, p-value $<0.001)$. Length showed most significant difference pre and post-operatively $(2.4 \pm 1.1$ vs. $1.3 \pm 1.1$, p-value $<0.001)$. Individual tumour extensions according to SIPAP for pre- and post-operative grading showed significant difference ( $p$-value $<0.001$ ), except for anterior extension. For suprasellar extension, $67.3 \%$ patients had pre-operative grade 3 and $63.3 \%$ had post-operative grade 0 . For infrasellar extension, $51.0 \%$ had pre-operative grade 2 and $71.4 \%$ had post-operative grade 0 . Anterior, posterior and parasellar extensions showed increased frequency in grade 0 in post-operative stage compared to pre-operative. Substantial inter-observer agreement was achieved for Superior, Inferior, Anterior and Posterior extent with all Kappa statistics values above 0.7 ( $p$ value $<0.001$ ).

\section{CONCLUSION}

We propose incorporating simple and objective SIPAP classification in routine MR reporting for ideal pituitary tumour delineation, relationship to juxtasellar structures and tumour size. 


\section{Background}

Pituitary lesions are associated with significant endocrinal abnormalities and need prompt management. The prevalence estimates of pituitary adenomas (PAs) are inconsistent. According to the most recent report from the Central Brain Tumor Registry of the United States, they account for approximately $16.9 \%$ of all brain tumors [1]. MR imaging is regarded as the modality of choice for the diagnosis of pituitary adenomas and there has been a rise in the detection of PAs with the widespread use of MRI. [2].

First line therapy for all the pituitary adenoma is surgical resection with exception of prolactinoma which shows excellent response to pharmacological therapy. [3] Transsphenoidal surgery is the approach of choice. [4] Compared with remission rates of $90 \%$ in microadenomas, macroadenomas with significant extrasellar extension have remission rates of $15-37 \%$ when treated with surgery alone. Radiation therapy and medical treatment often complement surgery. [5]

Simple and reproducible MR classification based on the major directions of tumour growth is an essential tool for surgical intervention. Careful evaluation of tumor extension on preoperative baseline MRI plays a vital role in providing the exact road map for the right surgical approach and maximum safe resection.[6]

SIPAP MR classification for pituitary adenomas [7] is derived from the KNOSP-STEINER classification [8] of parasellar growth, to which has been added a grading system for superior, inferior, anterior and posterior tumour extension.

We, therefore, propose to incorporate SIPAP classification in routine reporting template of pituitary adenomas, for ideal radiological description of tumour delineation, tumour relationship to juxtasellar structures, and tumour size (height, length and width), hence facilitating greater success rate in surgical and subsequent clinical management.

\section{Material And Methods}

\section{Patient Characteristics}

This study was a retrospective cross-sectional analysis of patients with pituitary macro adenomas who underwent pre and post-operative MRI brain with pituitary protocol between July 2019 to June 2020, interpreted by two experienced neuroradiologist with 8 and 3 years' experience (SS and AK) at our institution. Inclusion criteria were pre and post-operative MRI with pituitary protocol and histopathologically verified pituitary adenoma. Exclusion criteria were 1) Lack of preoperative imaging. 2) Prior medical therapy, radiation therapy, or chemotherapy, and 3) Inconclusive histopathology result. Based on these criteria, 49 out of 81 patients were enrolled in this study. The mean number of days between pre-operative and post-operative scan was 161 (minimum 1 day and maximum 971 days). This study was exempted and approved by the ethics review committee of our institution (EC No. 2020-495212875.)

\section{Image acquisition:}


MR studies performed on 1.5 and 3T superconducting system (Siemens and Toshiba respectively) with the use of a head coil. The acquisition matrix was $256 \times 256$ and the field of view $180 \mathrm{~mm}$. Sagittal TIweighted Spin Echo (SE) and coronal T1-weighted SE sequences before and after intravenous contrast administration acquired according to protocol.

\section{Grading system:}

The SIPAP classification was proposed by A. L. Edal et al in 1997. (Table 1). It is based on 6-figure number describing the extension of pituitary adenomas outside the sella. Grading number when read from left to right, there are 6 juxtasellar directions of interest: i) Suprasellar (0-4), ii) Infrasellar (0-2), iii) Parasellar right (0-4), iv) Parasellar left (0-4), v) Anterior (0-1) and 6. Posterior (0-1). Laterality of parasellar extension is taken as right or left and marked individually. T1 post contrast coronal MRI sequence is chosen for grading suprasellar and bilateral parasellar directions of tumour growth, while T1 post contrast sagittal images are used for infrasellar, anterior and posterior extensions. (FIGURE 1-5)

While reading the scans we made minor modification in posterior grading for improved clarification, that is, we further graded the posterior extension as posterior superior and posterior inferior (0-2). (FIGURE 4)

\section{Table 1: SIPAP classification based on the 6-figure registration.}

\begin{tabular}{|l|l|l|l|l|l|l|}
\hline & & \multicolumn{5}{c|}{ GRADING } \\
\hline S & \multicolumn{1}{|c|}{} & & & \\
\hline I & Suprasellar & 0 & 1 & 2 & 3 & 4 \\
\hline P Right & Infrasellar & 0 & 1 & 2 & & \\
\hline P Left & Parasellar Right & 0 & 1 & 2 & 3 & 4 \\
\hline A & Parasellar Left & 0 & 1 & 2 & 3 & 4 \\
\hline P & Anterior & 0 & 1 & & & \\
\hline
\end{tabular}

\section{Image interpretation:}

Post contrast T1 Coronal sections were assessed for suprasellar, infrasellar, right parasellar and left parasellar extension of tumor and post contrast T1 sagittal sections were reviewed for anterior and posterior extension of the tumor. Images were reviewed and results were recorded on two separate excel sheet for preoperative and post-operative MRI grading in 49 patients (total 98 scans) by each examiner individually and interobserver rating was calculated. The size of the pituitary tumor was assessed in three dimensions; height and width (craniocaudal and transverse dimensions respectively) were measured on coronal sections and length (anteriorposterior dimension) were measured on sagittal sections and subsequently volume of each tumor was calculated manually.

\section{Statistical Analysis:}


Data are presented as means with standard deviations for continuous variables of pre and postprocedure brain tumor characteristics (volume, height, length, width) of the patients, and tested for differences using paired t-test. Categorical variables such as patients' demographics and tumor extension grading are reported as frequency and percentages, tested using Chi-square or Fisher's exact test depending upon expected cell count distribution. Interobserver variability was calculated using Cohen's Kappa. A p-value of $<0.05$ was considered as cut off for significance. Data were analyzed using Stata version 15 (StataCorp, College Station, TX: StataCorp LLC.)

\section{Results}

Of the total 49 patients' data assessed for sellar tumor, $63.3 \%$ comprised of male with a mean age of 49 years (range 16 - 74) whereas $36.7 \%$ were of female gender with mean age of 47 years (range $24-66$ ).

Overall, the maximum preoperative volume was found to be $71.82 \mathrm{~cm} 3$ whereas the maximum postoperative (residual) volume was $49.50 \mathrm{~cm} 3$. Pre and post-operative pituitary tumor volumes were found to be significantly different $(14.1 \pm 17.7$ vs. $4.5 \pm 10.4$, p-value $<0.001)$. For separate volumetric parameters, length was found to be most significantly different for per and post-operative measurements (2.4 \pm 1.1 vs. $1.3 \pm 1.1$, p-value $<0.001)$. (Table 2 )

Individual assessment of tumor extensions was carried out according to SIPAP classification, each for pre and post-operative grading prevalence. Overall, all extensions were found statistically significantly ( $p$ value $<0.001$ ) for pre and post-operative stages except for the anterior extension. For Suprasellar extension, $67.3 \%$ of patient had pre-operative grade 3 and $63.3 \%$ had post-operative grade 0 . For Infrasellar extension, $51.0 \%$ had pre-operative grade 2 and $71.4 \%$ had post-operative grade 0 . Anterior, Posterior and Parasellar extensions also showed increased frequency in grade 0 in the post-operative stage as compared to the pre-operative stage. (Table 3)

High inter-observer agreement of tumor grading was achieved for Superior, Inferior, Anterior and Posterior extent for SIPAP classification. All Kappa statistics values were above 0.7 indicating substantial agreement with significant $p$-value of $<0.001$. Right and left Parasellar had moderate inter-observer agreement with Kappa statistic above 0.51 and 0.54 , having significant p-value of $<0.001$. (Table 4 ) 
Table 2

Pre and post-operative pituitary tumor volume

\begin{tabular}{|llll|}
\hline Brain Measurements $(\mathbf{c m})$ & $\begin{array}{l}\text { Pre-Brain } \\
(\text { Mean } \pm \text { SD) per cubic cm }\end{array}$ & $\begin{array}{l}\text { Post-Brain } \\
\text { (Mean } \pm S D) \\
\text { per cubic cm }\end{array}$ & P-value \\
\hline Volume* & $14.1 \pm 17.7$ & $4.5 \pm 10.4$ & $<0.001$ \\
\hline Height* & $2.8 \pm 1.3$ & $1.4 \pm 1.3$ & $<0.001$ \\
\hline Length* & $2.4 \pm 1.1$ & $1.3 \pm 1.1$ & $<0.001$ \\
\hline Width* & $2.8 \pm 1.3$ & $1.4 \pm 1.3$ & $<0.001$ \\
\hline *paired t-test & & & \\
\hline
\end{tabular}


Table 3

Extensions of the tumor and grading according to SIPAP classification

\begin{tabular}{|c|c|c|c|c|}
\hline Extensions of tumor & Grading & $\begin{array}{l}\text { Pre-operative } \\
N(\%)\end{array}$ & Post-operative N (\%) & P-value \\
\hline \multirow[t]{5}{*}{ Suprasellar } & 0 & $0(0.0)$ & $31(63.3)$ & \multirow[t]{5}{*}{0.00} \\
\hline & 1 & $6(12.2)$ & $4(8.2)$ & \\
\hline & 2 & $6(12.2)$ & $1(2.0)$ & \\
\hline & 3 & $33(67.3)$ & $12(24.5)$ & \\
\hline & 4 & $4(8.2)$ & $1(2.0)$ & \\
\hline \multirow[t]{3}{*}{ Infrasellar } & 0 & $21(42.9)$ & $35(71.4)$ & \multirow[t]{3}{*}{0.00} \\
\hline & 1 & $25(51.0)$ & $13(26.5)$ & \\
\hline & 2 & $3(6.1)$ & $1(2.0)$ & \\
\hline \multirow[t]{2}{*}{ Anterior } & 0 & $38(77.6)$ & $43(87.8)$ & \multirow[t]{2}{*}{0.10} \\
\hline & 1 & $11(22.5)$ & $6(12.2)$ & \\
\hline \multirow[t]{3}{*}{ Posterior } & 0 & $33(67.3)$ & 41(83.7) & \multirow[t]{3}{*}{0.00} \\
\hline & 1 & $12(24.5)$ & $5(10.2)$ & \\
\hline & 2 & $4(8.2)$ & $3(6.1)$ & \\
\hline \multirow[t]{5}{*}{ Paraseller Right } & 0 & 17(34.7) & $30(61.2)$ & \multirow[t]{5}{*}{0.00} \\
\hline & 1 & $12(24.5)$ & $7(14.3)$ & \\
\hline & 2 & $7(14.3)$ & $1(2.0)$ & \\
\hline & 3 & $8(16.3)$ & $6(12.2)$ & \\
\hline & 4 & $5(10.2)$ & $5(10.2)$ & \\
\hline \multirow[t]{5}{*}{ Paraseller Left } & 0 & $23(46.9)$ & $37(75.5)$ & \multirow[t]{5}{*}{0.00} \\
\hline & 1 & $13(26.5)$ & $3(6.1)$ & \\
\hline & 2 & $5(10.2)$ & $4(8.2)$ & \\
\hline & 3 & $4(8.2)$ & $1(2.0)$ & \\
\hline & 4 & $4(8.2)$ & $4(8.2)$ & \\
\hline
\end{tabular}


Table 4

Inter-observer Kappa statistics for SIPAP classification for tumor grading (pre-operative) ( $n=49)$

\begin{tabular}{|lllllll|}
\hline SUPERIOR & INFERIOR & ANTERIOR & POSTERIOR & $\begin{array}{l}\text { PARASELLAR } \\
\text { RIGHT }\end{array}$ & $\begin{array}{l}\text { PARASELLAR } \\
\text { LEFT }\end{array}$ \\
\hline Kappa & 0.769 & 0.771 & 0.766 & 0.724 & 0.512 & 0.545 \\
$\begin{array}{l}\text { p- } \\
\text { value }\end{array}$ & $<0.001$ & $<0.001$ & $<0.001$ & $<0.001$ & $<0.001$ & $<0.001$ \\
\hline
\end{tabular}

\section{Discussion}

HARDY'S classification was the first generally used radiographic classification of pituitary adenomas in which there was indirect assessment of suprasellar extension through pneumoencephalography [9]. This classification was not suitable for Computed tomography (CT) modality as uni direction of tumour growth was considered. However, from a neurosurgical perspective it was essential to delineate other extrasellar tumour extensions. Therefore, WILSON proposed modified grading system according to the degree of sellar destruction (grade) and extrasellar extension (stage) based on combined imaging and operative findings and emphasizing on tumor size and its extensions. This classification was helpful in designing therapy and had good prognostic value. [10]

With the advent of MRI, high resolution imaging and improved soft tissue contrast, a high degree of diagnostic accuracy was achieved [11]. In relation to reporting anatomical details and adequate topographical evaluation before surgical planning [12]. MRI post contrast coronal sections are the most helpful in demonstrating lateral extension of pituitary macroadenomas and its relation to adjacent parasellar structures [13] especially internal carotid arteries, which are most vulnerable to injure during surgery [13]. Much practical and simple grading system used till date was proposed by KNOSP to predict parasellar tumour growth into the cavernous sinus [8].

Later in 1997, A.L.Edal et al, proposed SIPAP classification which attempted to combine the KNOSPHARDY-WILSON classifications. With this combination they were able to offers a precise description of tumour growth in all directions, that is, lateral, upward and downward directions with the addition of forward and backward extensions. [7]

The SIPAP classification does not include tumor size, however it can be added as a supplement for recording and accurate reporting. LUNDIN \& PEDERSE in 1992 carried out a phantom study and proposed tumor volume assessment in three dimensions - width, length and height, and the formula given for this purpose was $0.5 \mathrm{x}$ width $\mathrm{x}$ length $\mathrm{x}$ height. [14] The tumor volume is important to note when there is a need to see the effect of pharmacological treatment of functioning adenomas. However, tumor extensions are more essential as a road map for surgical management and by default the tumor volume is meant to reduce postoperatively due to debulking. 
The SIPAP classification is based on post contrast coronal and sagittal TI-weighted MR images, which is a universally accepted method of imaging pituitary adenomas $[15,16]$ It has high diagnostic accuracy and reliability in assessment of tumor growth and useful tool in follow up. [17]

We had substantial agreement in assessing suprasellar, infrasellar, anterior and posterior extension of tumor in our study. However, there was moderate interobserver rating for parasellar extension which we believe is secondary to a fine line and minimal distance between the medial tangent, intercarotid section and lateral tangent. This aspect can be improved with experience.

This classification can be used for preoperative and postoperative tumor assessment as well as for pharmacologically treated tumors. In our study there was gross total resection (GTR) in 10 cases while rest of the 39 cases had maximum safe resection (MSR) with residual tumor.

We contemplate that the SIPAP classification can give neurosurgeons an indepth analysis of tumour extension and can predict expected complications during surgery.

Endoscopic transphenoidal approach, has a limited window, therefore, before embarking this surgical intervention, it is crucial to know the degree of parasellar extension to avoid injury to carotid arteries; extension of tumor anterior to the tubercle of sella or posteriorly behind the clivus because of their difficult approach; suprasellar extension as it may compress the optic chiasma and may obstruct foramen of Monro causing hydrocephalus; and last but not the least the inferior extension gives an idea of bony sella integrity.[11]

\section{Limitations}

This single-center study is limited by its nonrandomized and retrospective design. The application of SIPAP grading for pre-operative cases and post-operative cases with solitary remnant was straightforward. Post-operative cases with diverse remnants, however, cannot be classified based on this methodology due to ambiguity. Future prospective studies with large study cohort can also help propose a structured reporting format for interpreting pituitary adenomas.

\section{Conclusion}

Incorporation of SIPAP grading system in MRI reporting of pituitary adenomas is an effective and reliable tool to delineate tumour extension before treatment, predominantly surgical, providing an excellent road map for the surgeon and curtailing complications. Besides, it can be useful in follow up of tumour recurrence over time.

\section{Abbreviations}

MRI- Magnetic resonance imaging

PA- pituitary adenoma 
SE- Spin Echo

CT- Computed tomography

GTR-Gross total resection

MSR- Maximum safe resection

\section{Declarations}

\section{Ethics approval and consent to participate:}

This study was exempted and approved by the ethics review committee of Aga Khan University Hospital (EC No. 2020-4952-12875.)

Methods were carried out in accordance with Declaration of Helsinki

Consent for publication:

Not applicable

\section{Availability of data and materials}

The datasets used and/or analysed during the current study are available from the corresponding author on reasonable request.

\section{Competing interests}

The authors declare that they have no competing interests.

\section{Funding}

None

\section{Authors' contributions}

Idea perceived by SSMA and AK. KH and AA performed data collection and statistical analysis. SSMA and $A K$ read the images, prepared figures and tables. SSMA and AK wrote the main manuscript text with contribution from $\mathrm{KH}$ and $\mathrm{AA}$. All authors read and approved the final manuscript.

\section{Acknowledgements}

None

\section{References}


1. Matula C. Pituitary adenomas with invasion of the cavernous sinus space: a magnetic resonance imaging classification compared with surgical findings. Neurosurgery. 1993 Oct; 33(4):610-7.

2. \& VEZINAJ. L.: Transsphenoidal neurosurgery of intracranial neoplasm. In: Neoplasia in the central nervous system. Advances in neurology, vol. 15, p. 261. Edited by R. A. Thompson \& J. R. Green. Raven Press, New York 1976.

3. WILSONC . B.: A decade of pituitary microsurgery. J. Neurosurg. 61 (1984), 814.

4. Boxerman JL, Rogg JM, Donahue JE, Machan JT, Goldman MA, Doberstein CE. Preoperative MRI evaluation of pituitary macroadenoma: imaging features predictive of successful transsphenoidal surgery. AJR Am J Roentgenol. 2010 Sep;195(3):720-8.

5. Micko A, Oberndorfer J, Weninger WJ, Vila G, Höftberger R, Wolfsberger S, Knosp E. Challenging Knosp high-grade pituitary adenomas. J Neurosurg. 2019 May 31;132(6):1739-1746.

6. Lundin P, Bergström K. Gd-DTPA-enhanced MR imaging of pituitary macroadenomas. Acta Radiol. 1992 Jul;33(4):323-32.

7. Lundin P, Pedersen F. Volume of pituitary macroadenomas: assessment by MRI. J Comput Assist Tomogr. 1992 Jul-Aug;16(4):519-28.

8. Cottier JP, Destrieux C, Brunereau L, Bertrand P, Moreau L, Jan M, Herbreteau D. Cavernous sinus invasion by pituitary adenoma: MR imaging. Radiology. 2000 May;215(2):463-9.

9. Vieira JO Jr, Cukiert A, Liberman B. Magnetic resonance imaging of cavernous sinus invasion by pituitary adenoma diagnostic criteria and surgical findings. Arq Neuropsiquiatr. 2004 Jun;62(2B):437-43.

10. Dhandapani S, Singh H, Negm HM, Cohen S, Anand VK, Schwartz TH. Cavernous Sinus Invasion in Pituitary Adenomas: Systematic Review and Pooled Data Meta-Analysis of Radiologic Criteria and Comparison of Endoscopic and Microscopic Surgery. World Neurosurg. 2016 Dec;96:36-46.

\section{Figures}




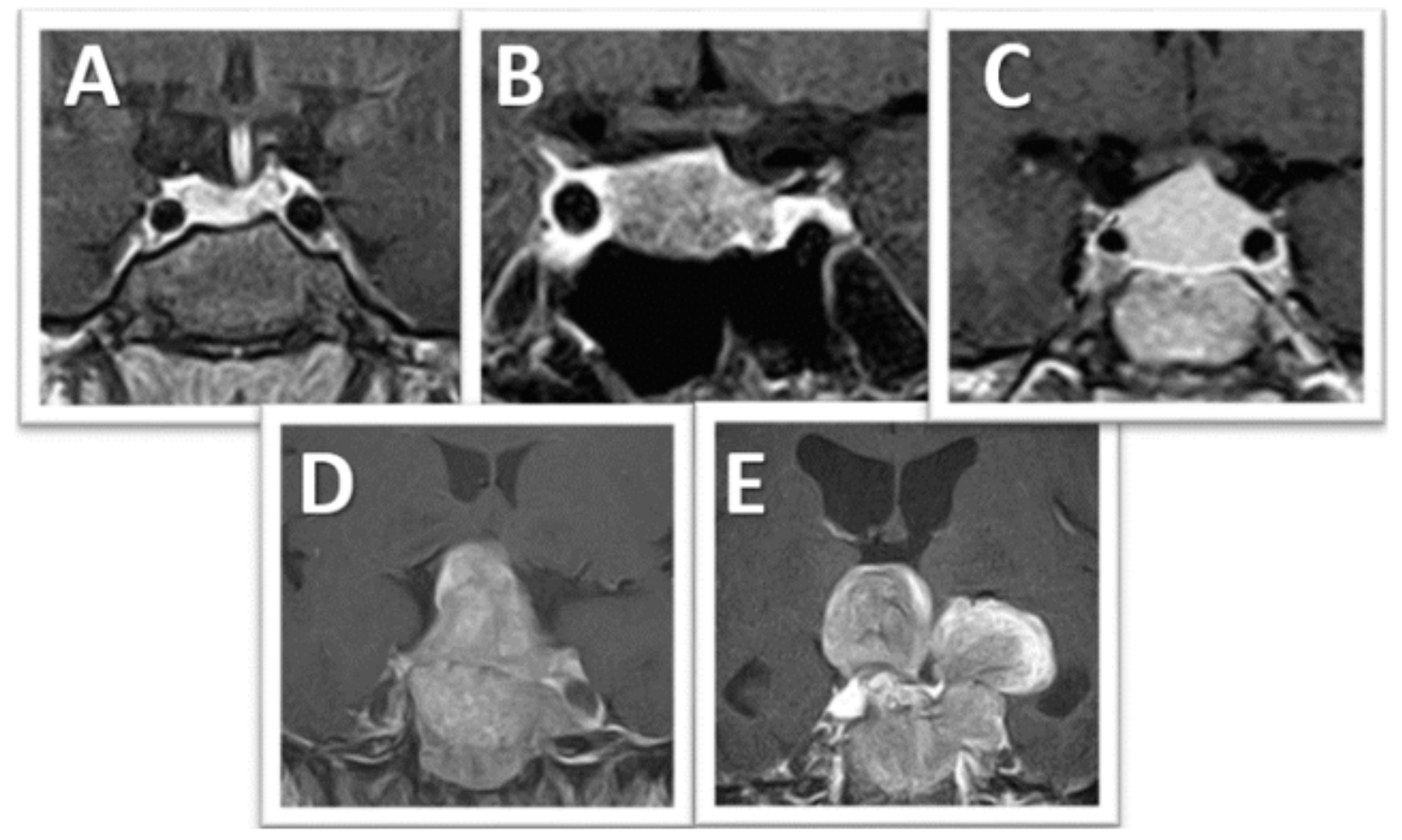

\section{Figure 1}

Coronal contrast enhanced T1 weighted MRI showing 5 grades of suprasellar extension. A) Grade 0: No bulge in the suprasellar space. B) Grade 1: Upward bulge of adenoma in suprasellar space without reaching the optic chiasm. C) Grade 2: Adenoma reaching optic chiasm but not displacing it. D) Grade 3: Adenoma displacing optic chiasm. E) Grade 5: Adenoma extension causing obstructive hydrocephalus.

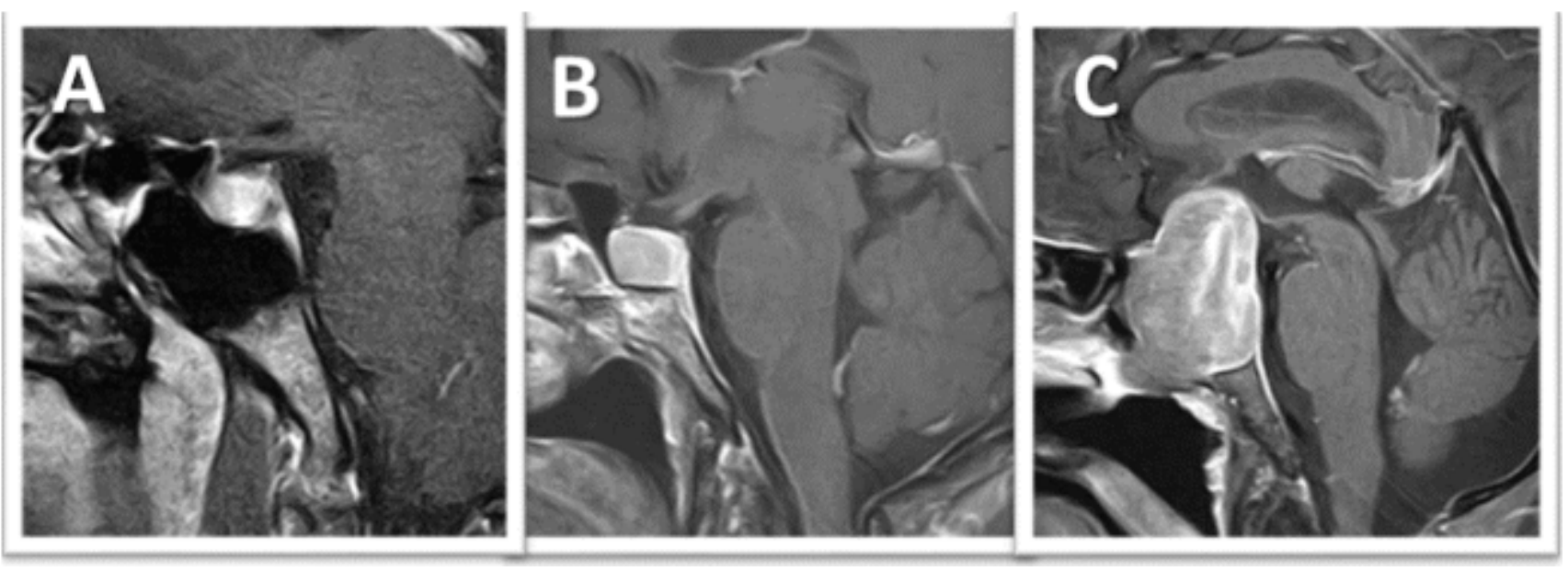

\section{Figure 2}

Sagittal contrast enhanced T1 weighted MRI showing 3 grades of infrasellar extension. A) Grade 0: No inferior bulge with intact floor of sella B) Grade 1: Focal inferior bulge of adenoma as an indirect sign of perforation of dura and floor of sella C) Grade 2: Adenoma extension beneath the sphenoid sinus. 

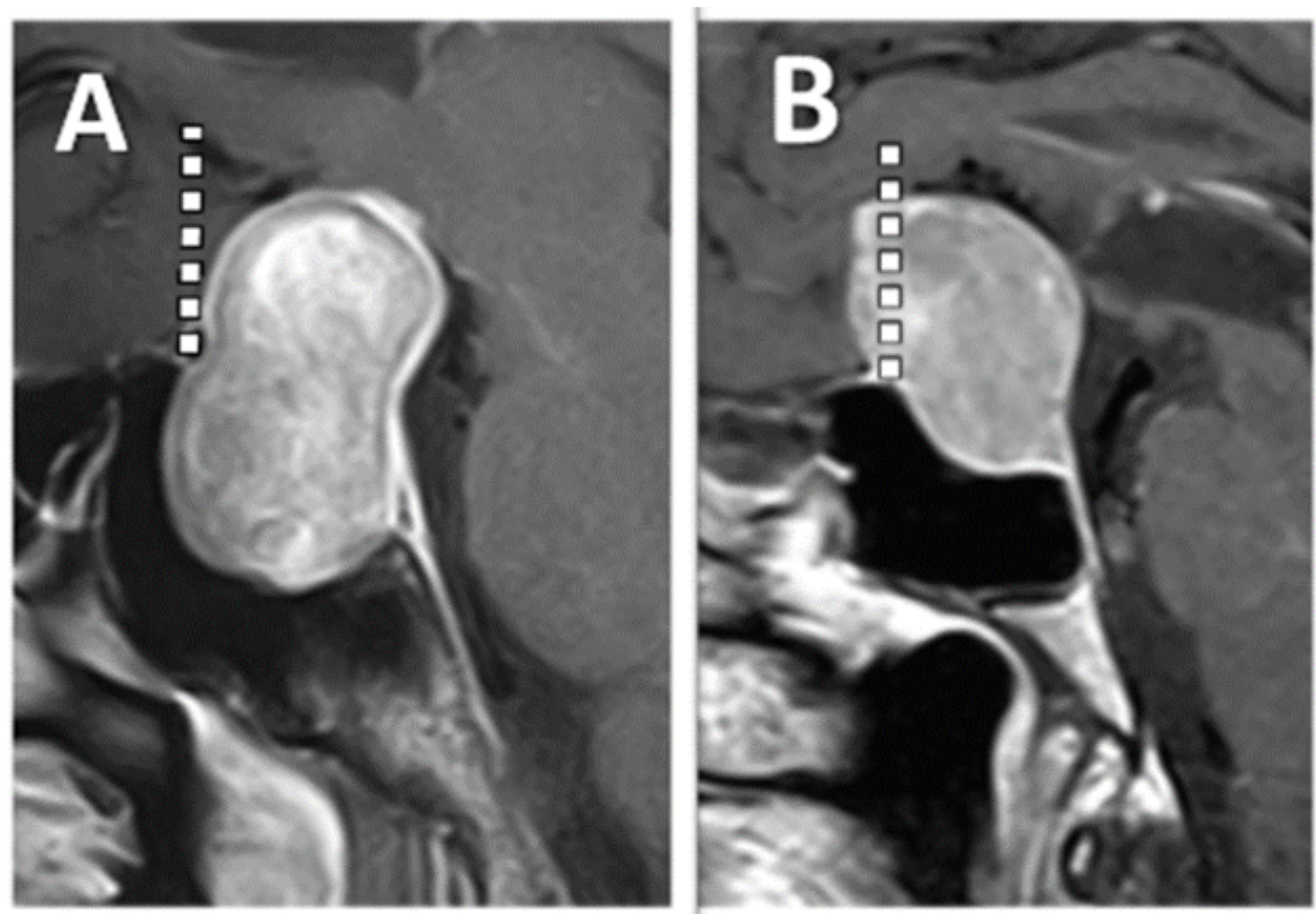

\section{Figure 3}

Sagittal contrast enhanced T1 weighted MRI showing 2 grades of anterior extension. Dotted line represents perpendicular line to the tubercle of sella A) Grade 0: No extension into anterior cranial fossa B) Grade 1: Extension into anterior cranial fossa
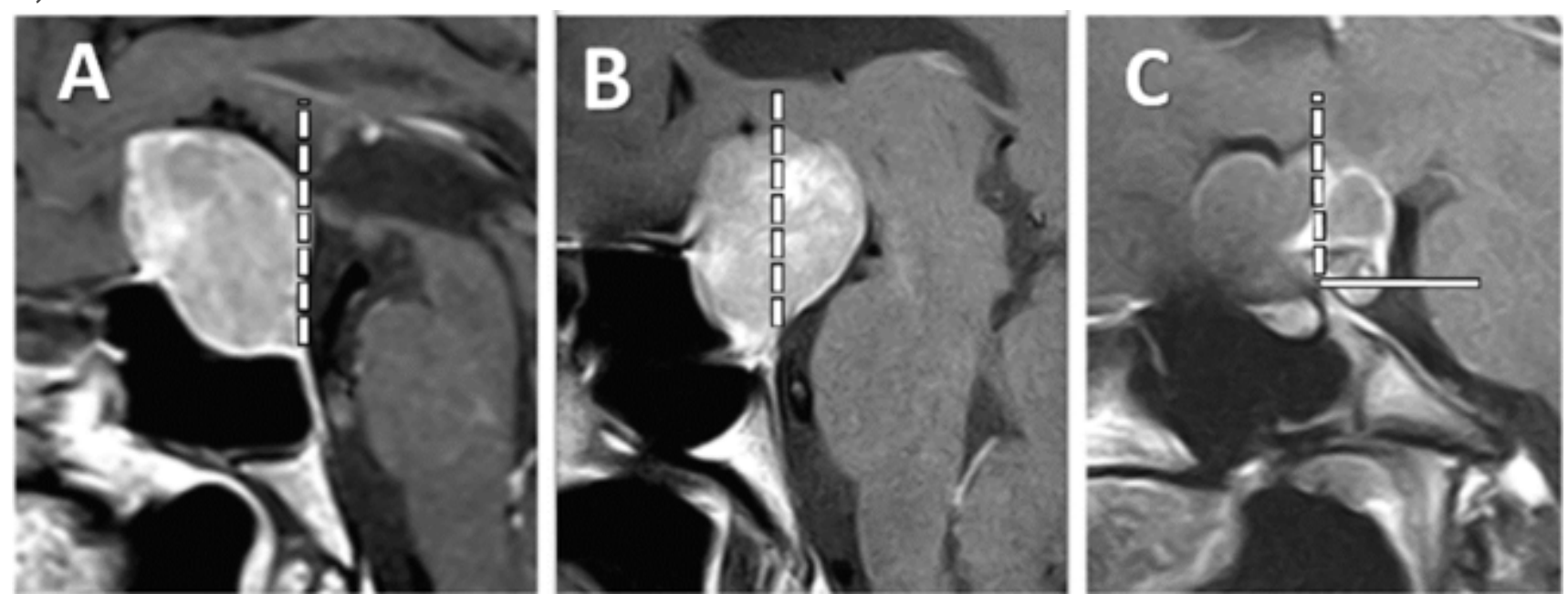

Figure 4

Sagittal contrast enhanced T1 weighted MRI showing 3 grades of posterior extension. Dotted line represents perpendicular line to the dorsum sella. Solid line represents horizontal line to dorsum sella A) 
Grade 0: No posterior extension B) Grade 1: Posterior-superior growth C) Grade 2: Posterior-inferior growth.
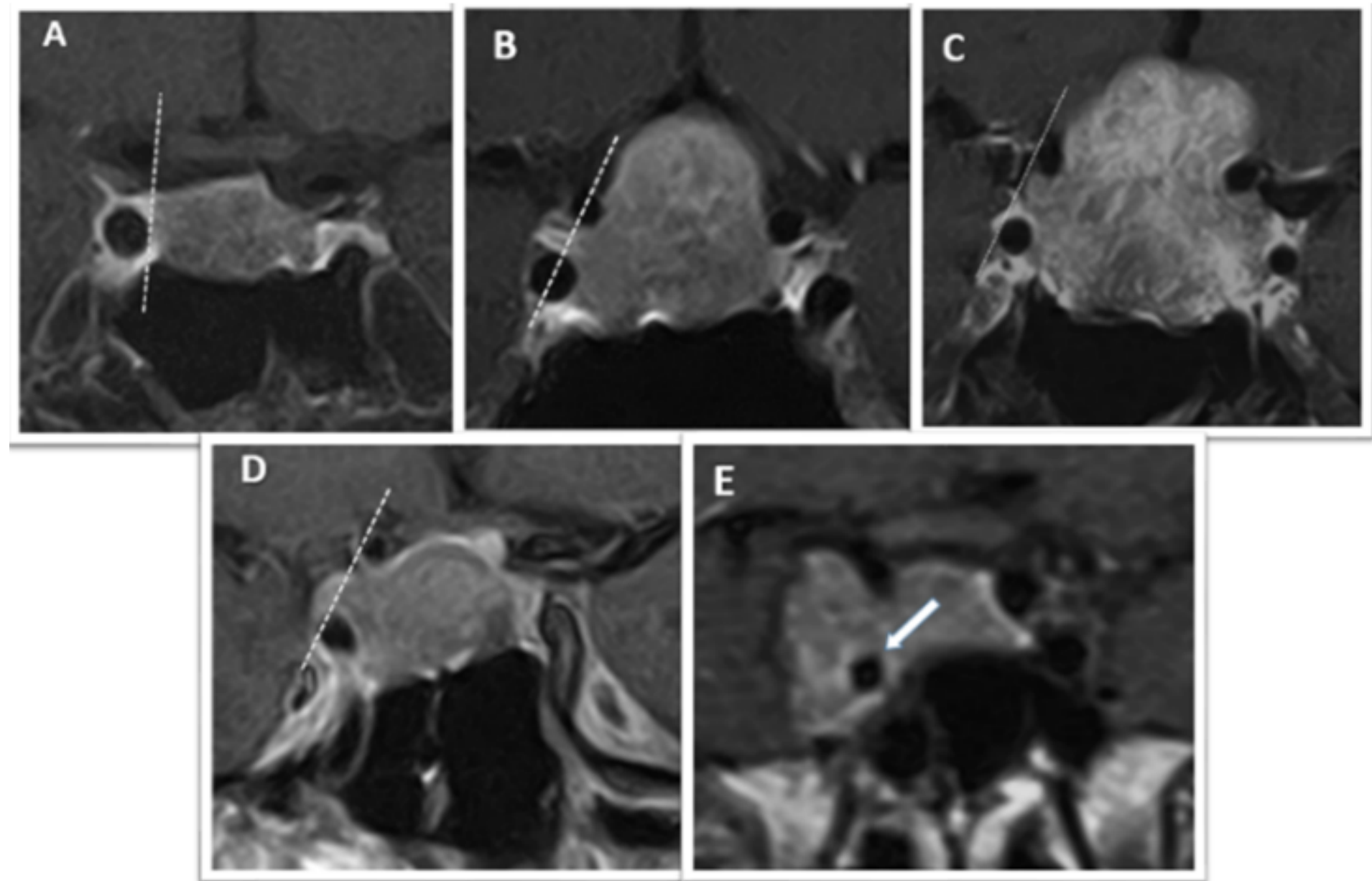

\section{Figure 5}

Coronal contrast enhanced T1 weighted MRI showing 5 grades of parasellar extension. Dotted lines drawn at the intra and supra cavernous internal carotid arteries A) Grade 0: Extension not beyond the medial tangent. B) Grade 1: Extension up to inter-carotid line. C) Grade 2: Extension up to lateral tangent. D) Grade 3: Extension beyond the lateral tangent E) Grade 4: Total encasement of intra-cavernous carotid artery.(solid arrow) 